\title{
Discussion about Application Effect of Orthopedic Rehabilitation Nursing on Patients Receiving Artificial Hip Replacement
}

\author{
Tan Qiaoling,*, You Yanjuan², Yu Xinhua ${ }^{1}$ \\ ${ }^{1}$ Rehabilitation Medical Center, Taihe Hospital affiliated to Hubei University of Medicine Hubei \\ Shiyan, 442000 \\ ${ }^{2}$ Department of Surgery, Zhushan County People's Hospital, Hubei, Zhushan, 442200 \\ *Corresponding author E-mail:6939940@qq.com
}

Keywords: artificial hip replacement; orthopedic; rehabilitation nursing

Abstract: Objective: to discuss the application effect of orthopedic rehabilitation nursing on patients receiving artificial hip replacement. Method: 68 patients receiving artificial hip replacement from January 2016 to December 2017 were extracted as the objects with the method of random number table. They were classified into 2 groups with double blind method. The patients in the control group received conventional nursing, while the patients in the observation group received orthopedic rehabilitation nursing. The clinical nursing situations of both groups were compared and analyzed. Results: rehabilitation excellence rate of observation group was $97.06 \%$, higher than the control group (76.47\%), $\mathrm{P}<0.05$. The complication occurrence rate of observation group was $5.88 \%$, lower than the control group (26.47\%), $\mathrm{P}<0.05$. Conclusion: the patients receiving artificial hip replacement show good effect after receiving orthopedic rehabilitation nursing, so it deserves clinical promotion.

Artificial hip replacement is mostly used to treat multiple hip joint diseases. In other words, artificial hip joint prosthesis is used to replace the diseased hip joint so as to reach the purpose of treating and repairing hip joint. This operation has been widely applied in recent years, and its development has been mature. However, the problem of postoperative complications fails to be handled effectively. With the rapid development of modern medicine, medical field has paid great attention to prevention and treatment of postoperative complications of artificial hip replacement. At present, corresponding nursing intervention is conducted for the patients receiving this operation to make the patients gain scientific and effective treatment and to play a positive role for prognosis improvement. To further improve rehabilitation effect of patients receiving artificial hip replacement, double blind method was applied to choose 68 patients receiving artificial hip replacement for control experiment so as to discuss the clinical effect of patients with artificial hip replacement receiving orthopedic rehabilitation nursing. 


\section{Data and method}

\subsection{General data}

68 patients receiving artificial hip replacement from January 2016 to December 2017 were chosen as the objects with the method of random number table. The disease situations of the patients were verified by pathological and imaging diagnosis, and they had typical clinical symptoms and received artificial hip replacement. The patients were classified into 2 groups with double blind method: control group (34 patients) and observation group (34 patients). There were 18 male patients and 16 female patients in the control group, with the age of 57-80 and mean value of $(68.09 \pm 5.14)$. There were 17 patients with new fracture of neck of femur, 9 patients with old fracture of neck of femur, 5 patients with aseptic necrosis of femoral head and 3 patients with rheumatoid coxitis. In the observation group, there were 19 male patients and 15 female patients, with the age of 54-83 and mean value of (68.72 \pm 5.53$)$. There were 16 new fracture of neck of femur, 10 patients with old fracture of neck of femur, 6 patients with aseptic necrosis of femoral head and 2 patients with rheumatoid coxitis. Clinical baseline data of both groups had no difference according to statistics software analysis, $\mathrm{P}>0.05$.

\subsection{Method}

The patients in both groups received artificial hip replacement. The patients in the control group received conventional nursing. The main content was to inform the patients of attentions, to help them correctly place the diseased limb, to pay close attention to the motion scope of hip joint and to warn the patients not to do strenuous exercise.

The observation group received orthopedic rehabilitation nursing. The main content is as follows: (1) psychological nursing. The relevant staff communicated with patients in time, assessed their psychological state, gave appropriate psychological counseling, informed the patients of impacts of negative emotion on the disease treatment, guided them to vent their mood, and introduced successful cases to the patients so as to make them confident in their recovery, improve their treatment compliance and cooperation, and make sure they face disease and treatment with positive attitude. (2) Health education. According to patients' cognition, the staff explained the knowledge about the disease to the patients, told them treatment mechanism and effect of artificial hip replacement, described the importance of functional training in the early stage, made sure the patients could face up to operative treatment and cooperate for rehabilitation treatment. (3) Rehabilitation exercise. The staff put a thick pillow between the patient's two legs to make the diseased limb extend $30^{\circ}$ outward and make the diseased hip less than $45^{\circ}$, and guided the patients to do rehabilitation training, such as lifting the leg straightly. 6h after the operation, the patients were guided to move on the bed, such as joint flexion and extension, and chest expansion exercise. In accordance with patients' disease improvement conditions, the optimal functional recovery training scheme was made for patients. In this period, exercise intensity intensified gradually, and the amount of exercise increased gradually. (4) Complication nursing. The staff taught the patients to correctly breathe and excrete sputum, helped the patients pat the back to excrete sputum so as to avoid pulmonary infection, and closely monitored patients' conditions. In case of stenocardia and hemoptysis, this indicated pulmonary embolism. Then, the visiting staff was immediately informed to handle it to avoid cardiac failure. The staff taught the patients to use the toilet stool, and helped them change their clothes, clean skin and turn over to avoid bedsore. 


\subsection{Observation indexes}

Functional recovery of hip joint in both groups was assessed, and various values were recorded. The occurrence rates of postoperative complications in both groups were figured out. Besides, contrastive analysis of various data was carried out.

\subsection{Evaluation criterion}

The follow-up visit was conducted for 3 months. Functional recovery of hip joint in both groups was assessed, and clinical rehabilitation effect was evaluated according to the results. Harris scoring was used to evaluate functional recovery of patients' hip joint before and after nursing. The score $\geq 90$ means excellent; the score 80 -89 means good; the score 60-79 means pass; the score $<60$ means poor ${ }^{[1]}$.

\subsection{Statistical method}

SPSS 20.0 statistical software was used to analyze various clinical data. The enumeration data were expressed with (\%), and tested with chi-square test. $\mathrm{P}<0.05$ means there is statistical significance.

\section{Results}

\subsection{Rehabilitation effect}

Rehabilitation excellence rate of observation group was $97.06 \%$, higher than the control group (76.47\%), $\mathrm{P}<0.05$, as shown in Tab.1.

Tab.1 Rehabilitation effect contrast of 2 groups $(\%, \mathrm{n})$

\begin{tabular}{|c|c|c|c|c|c|c|}
\hline Group & No. & Excellent & Good & Pass & Poor & $\begin{array}{c}\text { Excellence } \\
\text { rate (\%) }\end{array}$ \\
\hline $\begin{array}{c}\text { Observation } \\
\text { group }\end{array}$ & 34 & 20 & 13 & 1 & 0 & $\begin{array}{c}33 \\
(97.06 \%)\end{array}$ \\
\hline Control group & 34 & 11 & 15 & 7 & 1 & $\begin{array}{c}26 \\
(76.47 \%)\end{array}$ \\
\hline$X^{2}$ value & - & - & - & - & - & 6.2750 \\
\hline P value & - & - & - & - & - & 0.0122 \\
\hline
\end{tabular}

\subsection{Complications}

The complication occurrence rate of observation group was $5.88 \%$, lower than the control group (26.47\%), $\mathrm{P}<0.05$, as shown in Tab.2.

Tab.2 Complication contrast of 2 groups $\quad(\%, n)$

\begin{tabular}{|c|c|c|c|c|c|c|}
\hline Group & No. & Dislocation & Infection & \multicolumn{2}{|c|}{$\begin{array}{c}\text { Pressure sores } \\
\text { Deep venous } \\
\text { thrombosis }\end{array}$} & $\begin{array}{c}\text { Total occurrence } \\
\text { rate (\%) }\end{array}$ \\
\hline $\begin{array}{c}\text { Observation } \\
\text { group }\end{array}$ & 34 & 0 & 1 & 0 & 1 & $2(5.88 \%)$ \\
\hline Control group & 34 & 2 & 3 & 1 & 3 & $9(26.47 \%)$ \\
\hline $\mathrm{X}^{2}$ value & - & - & - & - & - & 5.3142 \\
\hline P value & - & - & - & - & - & 0.0212 \\
\hline
\end{tabular}




\section{Discussion}

After artificial hip replacement, the probability of prosthetic loosening, dislocation, infection or phlebothrombosis is very large, and these will affect patients' joint functional recovery and seriously affect their living quality ${ }^{[2][3[4]}$. In order to further promote clinical treatment effect of artificial hip replacement and fully improve patients' prognosis, scientific and appropriate nursing intervention should be conducted for the patients.

Modern medical research has proven that, the patients with hip replacement have obvious effect after receiving systematic rehabilitation nursing in the perioperative period, and rehabilitation nursing can promote clinical treatment effect, accelerate patients' blood circulation, improve their muscle strength, provide a favorable environment for hip joint functional recovery and play a significant role in improving their living quality ${ }^{[5][6]}$. There have some research results show that, rehabilitation nursing intervention have positive influence on rehabilitation effect and self-care ability of patients receiving total hip replacement ${ }^{[7]} .1$ month after the operation, Harris score of patients was higher than that of control group, and the treatment excellence rate was $87.50 \%$. In addition, Barthel index was about 72 . Hence, their daily living ability improved significantly, and the improvement degree was superior to the control group. Rehabilitation nursing is a new nursing intervention mode. The nursing content is the specialized nursing technology based on the conditions of the disease. The nursing aims to let patients get scientific and comprehensive nursing intervention, improve their clinical treatment effect and significantly improve their prognosis ${ }^{[8]}$. After the patients receive hip joint replacement, large operative wound will usually be left. Thus, the probability of multiple complications is large after the operation. Healing deformity is easily seen clinically. Some patients are anxious, because they do not understand the operation. Thus, their psychological state is not stable, which affects their treatment enthusiasm and thus affects their recovery. Therefore, scientific and appropriate psychological intervention is required to eliminate patients' negative emotion, make their psychological state stable and ensure smooth implementation of clinical treatment ${ }^{[9]}$. Meanwhile, the state of the disease and treatment knowledge should be explained for patients to make them further understand their disease and overall cognize artificial hip replacement so as to actively cooperate for the clinical treatment and rehabilitation exercise. Besides, this can make sure postoperative rehabilitation proceeds orderly, avoid functional exercise delay after the operation and avoid the impacts on the functional recovery ${ }^{[10]}$. If patients carry out functional exercise early after the operation, their blood circulation will improve, and phlebothrombosis can avoid. In addition, the probability of tissue adhesion declines. Thus, they can fully improve their muscle force and enhance joint stability. The occurrence rate of postoperative complication also decreases ${ }^{[11]}$. In this study, the patients in the observation group received orthopedic rehabilitation nursing. The nursing content included psychological nursing, rehabilitation guidance, health education, functional exercise and complication nursing etc. Patients' state of disease, and physical and psychological health were taken into account. The results show that, rehabilitation excellence rate of patients in the observation group was $97.06 \%$, higher than the control group (76.47\%). Moreover, the occurrence rate of postoperative complications was 5.88\%, lower than the control group (26.47\%), $\mathrm{P}<0.05$. This indicates that, the patients with artificial hip replacement have significant clinical effect after receiving orthopedic rehabilitation nursing intervention. Such nursing intervention plays a positive role in improving the disease and reducing complications.

In conclusion, orthopedic rehabilitation nursing has obvious effect for the patients receiving artificial hip replacement. Orthopedic rehabilitation nursing can promote patients' rehabilitation effect, and reduce patients' occurrence rate of rehabilitation efficacy. Therefore, it has optimal promotion value. 


\section{References}

[1] Mariana Kátia Rampazo-Lacativa, Maria José D’Elboux.Effect of cycle ergometer and conventional exercises on rehabilitation of older patients with total hip arthroplasty: study protocol for randomized controlled trial.Trials. 2015; 16: 139. Published online 2015 Apr 8. doi: 10.1186/s13063-015-0647-8.

[2] Marie D Westby, Catherine L Backman.Patient and health professional views on rehabilitation practices and outcomes following total hipand knee arthroplasty for osteoarthritis:a focus group study.BMC Health Serv Res. 2010; 10: 119. Published online 2010 May 11. doi: 10.1186/1472-6963-10-119

[3] Georgios I. Drosos, Athanasios Ververidis, Christos Valkanis, Grigorios Tripsianis, Eftihios Stavroulakis, Theodosia Vogiatzaki, Konstantinos Kazakos.A randomized comparative study of topical versus intravenous tranexamic acid administration in enhanced recovery after surgery (ERAS) total knee replacement.J Orthop. 2016 Sep; 13(3): $127-131$. Published online 2016 Mar 26. doi: 10.1016 /j.jor. 2016.03.007

[4] Christopher P. Childers, Anaar E. Siletz, Emily S. Singer, Claire Faltermeier, Q. Lina Hu, Clifford Y. Ko, Gregory J. Golladay, Stephen L. Kates, Elizabeth C. Wick, Melinda Maggard-Gibbons.Surgical Technical Evidence Review for Elective Total Joint Replacement Conducted for the AHRQ Safety Program for Improving Surgical Care and Recovery.Geriatr Orthop Surg Rehabil. 2018; 9: 2151458518754451. Published online 2018 Feb 12.doi: 10.1177/ 2151458518754451

[5] EL Jones, TW Wainwright, JD Foster, JRA Smith, RG Middleton, NK Francis.A systematic review of patient reported outcomes and patient experience in enhanced recoveryafter orthopaedic surgery.Ann R Coll Surg Engl. 2014 Mar; 96(2): 89-94. Published online 2014 Mar.doi: 10.1308/ $003588414 X 13824511649571$

[6] Ajay Malviya, Kate Martin, Ian Harper, Scott D Muller, Kevin P Emmerson, Paul F Partington, Mike R Reed.Enhanced recovery program for hip and knee replacement reduces death rate: A study of 4,500 consecutive primary hip and knee replacements.Acta Orthop. 2011 Oct; 82(5): 577-581. Published online 2011 Nov 24. doi: 10.3109/17453674.2011.618911

[7] JF Maempel, PJ Walmsley.Enhanced recovery programmes can reduce length of stay after total knee replacement without sacrificing functional outcome at one year.Ann R Coll Surg Engl. 2015 Nov 1; 97(8): 563-567. Published online 2015 Nov 1. doi: 10.1308/ rcsann. 2015.0016.

[8] Eva N Glassou, Alma B Pedersen, Torben B Hansen.Risk of re-admission, reoperation, and mortality within 90 days of total hip and knee arthroplasty infast-track departments in Denmark from 2005 to 2011.Acta Orthop. 2014 Sep; 85(5): 493-500. Published online 2014 Sep 5. doi: 10.3109/17453674. 2014.942586.

[9] Prasad Antapur, Nizar Mahomed, Rajiv Gandhi.Fractures in the elderly: when is hip replacement a necessity? Clin Interv Aging. 2011; 6: 1-7. Published online 2010 Dec 20. doi: 10.2147/ CIA.S10204

[10] Health Quality Ontario.Physiotherapy Rehabilitation After Total Knee or Hip Replacement: An Evidence-Based Analysis.Ont Health Technol Assess Ser. 2005; 5(8): 1-91. Published online 2005 Jun 1.

[11] Christopher P. Childers, Anaar E. Siletz, Emily S. Singer, Claire Faltermeier, Q. Lina Hu, Clifford Y. Ko, Gregory J. Golladay, Stephen L. Kates, Elizabeth C. Wick, Melinda Maggard-Gibbons.Surgical Technical Evidence Review for Elective Total Joint Replacement Conducted for the AHRQ Safety Program for Improving Surgical Care and Recovery.Geriatr Orthop Surg Rehabil. 2018; 9: 2151458518754451. Published online 2018 Feb 12.doi: 10.1177/ 2151458518754451. 